\title{
Muscular wasting, sarcopenia and cachexia: a trouble for the patients, a challenge for the doctors. The role of the person in this dramatic scenario
}

\author{
Evasio Pasini
}

Received: 22 October 2012/Accepted: 15 November 2012/Published online: 11 December 2012

(C) Springer-Verlag Italia 2012

\begin{abstract}
Nowadays, the importance of muscle mass, strength, and metabolic function is demonstrated in both the daily life activities and prognosis. However, the evaluation of muscle mass is not routinely measured by doctors. Clinically, the loss of muscular mass is classified as: muscular wasting, sarcopenia, cachexia. The pathogenesis of sarcopenia-wasting-cachexia is multi-factorial. Indeed, imbalance between anabolic and catabolic signaling, named as "hypermetabolic syndrome", is the fundamental cause of muscular loss. The evaluation of muscular metabolic impairment should consider several aspects. Body mass index (BMI) is a good first step to use. However, BMI cannot distinguish lean muscular from fatty mass. Therefore, muscle and global catabolic/anabolic metabolism can be measured by: (a) anthropometric measurements, (b) dualenergy X-ray absorptiometry (DEXA or DXA), visceral proteins as blood albumin, transferrin, prealbumin, (c) total lymphocytes blood count, (d) nitrogen balance. Several therapeutic strategies have been proposed to cure muscle loss. Recent works showed that specific mixtures of aminoacids, including essential one, calculated according to stoichiometric ratio, counteract sarcopenia. Other intriguing approaches to muscular loss have been recently proposed. A recent trial showed that Amalirin can reverse muscle wasting in cancer cachexia. In addition, anabolic process can be also modulated by stimulation of selective androgen receptor modulators in humans and by activation of activine type II receptor of Myostatin in both animal models and humans. One other animal study shows that anabolic/
\end{abstract}

E. Pasini $(\bowtie)$

Rehabilitative Cardiology Division, Institute of Lumezzane, Fondazione Salvatore Maugeri, IRCCS, Via Giuseppe Mazzini 129, 25066 Lumezzane (Brescia), Italy

e-mail: ev.pasini@gmail.com catabolic transforming agent MT-102 reverses muscle wasting in rat. Muscular wasting, sarcopenia and cachexia are conditions with important clinical, social and economic impact. However, they are often underestimated by the patients and/or not properly evaluated and cured by physicians. Specific work must be planned and organized to sensitize the "person" on estimating and maintaining the muscle mass and its metabolic and functional roles since they are fundamental in both healthy people and ill patients.

Keywords Muscular wasting - Sarcopenia . Cachexia amino acid

\section{The clinical and cultural problem}

The importance of muscle mass, strength, and metabolic function in the performance of exercise and in the daily life activities has never been questioned. Until several years ago, the muscle was considered a structure fundamental only for the body movement. Although nowadays research shows that muscle plays a pivotal role in the regulation of body metabolism, patients and a great number of doctors continue to consider the muscle (and the clinical impairment associated) as an organ responsible for movement underappreciating and underestimating its role in health and diseases. [1] Indeed our understanding on the metabolic role of muscle in the body life is increasing day by day. Evidences show that muscle is fundamental for maintenance of protein and glucose metabolism. Many hormone receptors are located in the muscle and regulate body anabolism and catabolism. In addition, muscle is metabolically linked with liver, kidney, fat tissue and lung for the production of metabolic intermediates, fundamental for body life. Notably, muscle loss increases mortality and 
morbidity. [2] Indeed, as early as in the third century BC, the Greek physician Hippocrates from Kos very neatly described the muscular wasting syndrome associated with terminal disease. "The flesh is consumed and becomes water,...the abdomen fills with water, the feet and the legs swell, the shoulders, clavicles, chest and thighs melt away. This illness is fatal." The possible effects of the impaired protein metabolism with muscle loss are shown in Table 1 [3].

However, many patients do not know the importance of the maintenance of an adequate muscular mass and function. Often, the reduction of body weight, reached with specific diet, is considered an appreciable event. We do not believe that the majority of patients (and probably of many

Table 1 The possible effects of impaired protein metabolism with muscle loss

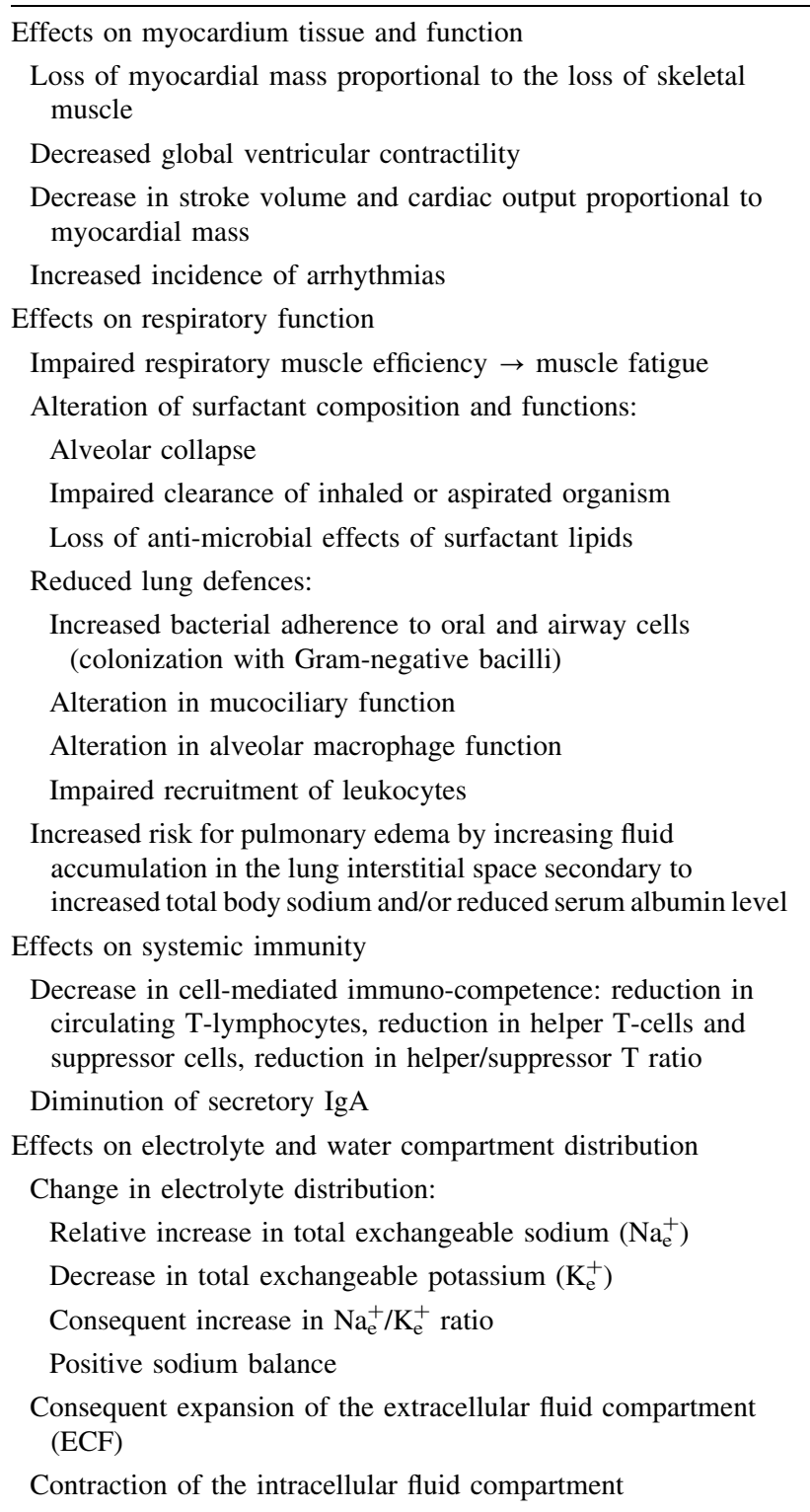

doctors) know that body weight is the result of water, fat and lean (muscular) mass and that reduction of weight often corresponds to reduction of water and lean mass but not of fat tissue with negative influences on global body metabolism. Consequently, the lean mass evaluation is fundamental for a personalized patient's therapy to maintain an adequate muscle mass after a diet and, more importantly, during critical ill or chronic diseases as diabetes, heart failure, renal failure, cancer, respiratory insufficiency, obesity, and autoimmune disease as well as in a para-physiological condition as senescence. Indeed, we have recently shown in a geriatric intensive rehabilitation centre that the simple maintenance of muscle and visceral protein metabolism reduces the risk of infection by $30 \%$ [4]. However, until now, the evaluation of muscle mass is performed during a medical visit only in specific medical centers, but it is not routinely measured.

\section{The clinical classification}

Muscular wasting with consequent sarcopenia and cachexia is frequent in older people and in patients with chronic diseases. Often, the cause responsible of the muscle mass loss is indistinguishable in clinical setting. However, a recent paper tries to distinguish beyond the semantics of the words sarcopenia and cachexia [5].

The term sarcopenia is derived from the Greek words sarx (flesh) and penia (poverty). Sarcopenia is characterized by loss of muscle mass and strength with consequent loss of autonomy. Sarcopenia is multifactorial and can have different origins. It is defined as age-related or "primary" when age solely can explain the muscle loss, and function or "secondary" when it is related or associated to one or more causes as sedentary lifestyle, malnutrition, deconditioning, organ failure, and inflammatory diseases. Several criteria for diagnosis of sarcopenia have been proposed. The evaluation of muscle mass and strength is recommended [5].

The term cachexia is derived from the Greek words kakos (bed) and héxis (condition). It is defined as a "complex metabolic syndrome associated with underlying illness and characterized by loss of muscle mass with or without fat mass". Cachexia is defined as weight loss of at least $5 \%$ of body weight in the previous 12 months [5]. It is associated with a change in body composition with body weight decrease due to loss of lean and fat mass. In advanced cachexia, ipoalbuminemia is also present as result of an advanced and severe impairment of the protein and global metabolism.

Interestingly, we have to introduce the concept of sarcopenic obesity. Indeed, the current definition of obesity is based on the body mass index (BMI) and does not take into 
account the body composition and the ratio lean/fat mass which regulates the body metabolism. So, sarcopenic obesity can be defined as increased BMI but with reduced lean body mass and function.

\section{Why do muscular wasting, sarcopenia and cachexia occur?}

The pathogenesis of the syndrome sarcopenia-wastingcachexia is multi-factorial. Identification of predominant alterations of metabolism may be the key for interpreting each patient's predominance of factor(s), although we think that the galaxy of events ascribed to a "hypermetabolic syndrome" finally drives to a unique path of imbalance between anabolism and catabolism.

Indeed, both elderly sarcopenia and more pronounced ill patients with cachexia have modifications of balance of circulating anabolic molecules (i.e., insulin, insulin like growth factors, growth hormone, others) and catabolic ones (i.e., TNF-Alfa, cortisol, catecholamines, glucagons, cytokines as interleukins, others.) which alter the ratios between anabolism and catabolism. This pattern is identified as "hypercatabolic syndrome" (HS) and causes significant changes in both muscular and global metabolism [6].

In normal skeletal muscle, the continuous turnover of proteins is a basic process of cell life. Data show that, in healthy human individuals, about $250-350 \mathrm{~g}$ of protein per day is degraded in the muscles. Some of the amino acids (AAs) produced are re-used from the cells for de novo protein synthesis or to produce energy-rich intermediates for oxidation via the Krebs cycle and the respiratory chain. However, a large quantity of AAs are released into the blood to maintain the blood pool of AAs. The balance between muscle protein synthesis and breakdown determines the overall cell protein content and metabolism. The process removes unnecessary proteins and provides the raw material for the synthesis of new proteins or for energy metabolism through oxidation of AAs. Interestingly, protein synthesis and degradation is regulated by the balance of catabolic and anabolic stimuli. The increase of catabolic hormones and/or molecules and the reduction of anabolic hormone co-operate to the HS characterized by intensive muscular cellular proteolysis and AAs release with a number of metabolic consequences including insulin resistance, reduced cytoplasmic and mitochondrial cell protein synthesis and impaired cell functions and energetic metabolism. AAs are used not for cellular protein replacement and/or cellular energy production but for maintenance of the global metabolism of the mammalian organism [6].

In fact, the carbon skeleton, which originates from deamination of AAs released from skeletal muscle, is used in the liver to produce glucose by gluconeogenesis and other fundamental molecules for the body life. Interestingly, the intake of cell glucose is reduced in the muscular wasting syndrome. This phenomenon is named "insulin resistance" and it is due to hormonal impairments. However, glucose is essential to maintain the glucose-dependent metabolism of fundamental structures such as the brain and erythrocytes and consequently is over-synthesised from AAs in HS [6].

The impairment of protein muscle and global metabolism in a chronic disease as chronic heart failure (CHF) has recently been demonstrated. We noted that patients with CHF have important massive muscle proteolyses with consequent muscular proteolysis and AAs release while performing light daily type exercise. Indeed, light exercise causes muscle lean mass loss and impairment of global metabolism [7].

\section{How to identify muscular wasting, sarcopenia and cachexia?}

The ideal marker to identify muscular wasting does not exist.

We believe that it is important to integrate patient's clinical information with specific laboratory markers.

The evaluation of BMI is a good first step to assess metabolic impairment because $85 \%$ of the ideal body weight is an index of protein-calorie impairment. However, BMI is only partially useful because it cannot distinguish lean muscular from fatty mass.

Therefore, global and muscle protein metabolism can be measured by the following biomarkers [8]:

1. Anthropometric measurements: they allow a qualitative and qualitative analysis of body composition, distinguishing muscular lean mass from fatty mass.

Dual-energy X-ray absorptiometry (DEXA or DXA) is widely used for qualitative and quantitative analysis. However, DXA scanners are expensive equipment and although DXA uses low energy X-ray, patients are still exposed to radiation.

In addition, computerized tomography (CT scan) is used to evaluate lean and fat tissues. CT scan combines $\mathrm{X}$-ray images with the aid of a computer, to generate cross-sectional view or three dimensional images. It is repeatable and relatively inexpensive. However, CT scan exposes patients to X-rays. For this reason it is often used to study simultaneously both internal organs and body composition in patients with concomitant diseases as cancer.

Also impedentiometry is useful for anthropometric measurements but in patients without water retention. Simpler, less expensive and non-invasive methods of 
estimating body composition as indirect evaluation of general protein metabolism include the development of skinfold thickness (TST)_measured by a plicometer-as an index of fatty mass and arm muscle area (AMA) - measured in centimeters - as an index of lean muscular mass. Both of them can be evaluated routinely at the pa tient's bedside.

2. Visceral proteins: quantification of blood albumin, transferrin, prealbumin, retinol binding protein provides information on protein synthesis and metabolism.

3. Total lymphocyte blood count: it is an indirect index of cell proliferation, protein synthesis and energy availability. It provides information on global and protein metabolic impairment.

4. Nitrogen balance: it measures the dynamic protein metabolism. It is the ratio between the nitrogen coming from AAs of food we eat and the quantity of nitrogen excreted from the urine which originates from protein catabolism.

\section{How to treat muscular wasting, sarcopenia and cachexia?}

Treatment of muscular wasting is not well established yet. Indeed, dedicated guidelines are not available.

However, treatment of sarcopenia and cachexia should be started concomitantly with the cure of the possible disease underlying the muscle loss aiming at improving results in terms of patient's mortality, morbidity and quality of life. To reach these aims, the identification of molecular and metabolic mechanism responsible for sarcopenia and cachexia is fundamental.

Nowadays, the most studied treatment is the nutritional one.

Indeed, intake and blood availability of AAs are a key factor for maintaining cellular and general metabolism, muscle proteins synthesis and muscle mass of mammals. We have to remember that AAs in the diet are absorbed, after protein digestion, by pancreatic enzymes. However, the pancreas uses large amounts of AAs and energy to produce digestive enzymes [6].

In HS the efficiency of pancreas and mesenteric circulation may be progressively reduced. These conditions lead to impaired AAs digestion and absorption and consequently to reduced AAs plasma patterns which may therefore be insufficient to maintain the protein synthesis and energetic needs of HS patients [6]. So, increase in protein intake with meal can not increase muscle protein synthesis, accordingly.

On the contrary, individual AAs in nutritional supplements are not digested. They are rapidly absorbed and therefore immediately available in the bloodstream and transported into the cells [6].

In addition to that, nowadays we know that specific AAs have multiple metabolic roles beyond simple substrate for protein synthesis. Indeed, essential amino acids (EAAs) stimulate protein synthesis in both young and elderly people [9].

Moreover, it has been recently demonstrated that a specific mixture of individual AAs, including essential ones, calculated according to stoichiometric ratio, contains either those aminoacids necessary for: (1) paying energetic needs, (2) maintaining protein synthesis, and (3) stimulating mitochondrial biogenesis [10].

This is possible because some specific AAs control protein synthesis in myocytes by activation of AMP-activated protein kinase (AMPK) and mammalian target of rapamicin (mTOR) which are important enzymes able to regulate both energy production/utilization, protein synthesis, cell proliferation, mitochondrial biogenesis and antiapoptotic process. All these actions are anabolic ones and counteract the catabolic stimulation by hormones and cytokines [11].

Clinical and experimental data suggest that exogenous oral supplements with specific mixture of individual AAs, administered with traditional therapy, counteract muscular wasting and cellular energy reduction in chronic diseases as CHF improving muscle performances and therefore the patient's quality of life [12].

The practical clinical consequence of these observations is that instead of nitrogen provided by diet, caloric and nitrogen needs should be calculated separately considering the amount of amino acids able to stimulate specific metabolic pathways which maintain muscular and body metabolism integrity [13].

This knowledge can explain why a previous old study did not demonstrate any effect of the protein dietary supplement on the muscle metabolism [14].

In light of these observations, Layman has said recently that the new version of the dietary guidelines for Americans has to consider the results of new research and should contain the following new concepts [13].

- Protein and individual AAs are critical part of the adult diet

- Protein and individual AAs needs are proportional to the body weight and not to the energy intake

- Most adults benefit from protein and individual AAs intake above the minimum recommended daily allowance (RDA)

Other intriguing approaches to muscular loss have been recently proposed.

A recent multicentre phase III clinical trial showed that Amalirin (orally active ghrelin receptor antagonist) can 
reverse muscle wasting in cancer cachexia and was proposed as therapeutical agent in all other stage of cachexia.

Anabolic process can be also modulated by stimulation of selective androgen receptor modulators (SARMs) in humans and by activation of the activine type II receptor of Myostantin in both animal models and humans.

Other animal study shows that anabolic/catabolic transforming agent MT-102 reverses muscle wasting in rat [15].

Physical activities, as non-pharmacological intervention, improve muscle mass and strength and can be recommended in sarcopenic and cachectic patients concomitantly with other therapeutical strategies. Particularly, aerobic exercise increases mitochondrial number and metabolical activities, while resistance exercise meliorates muscle mass and strength increasing proteins synthesis and fiber size [16].

\section{Conclusion}

Muscular wasting, sarcopenia and cachexia are conditions with important clinical, social and economic impacts. However, they are often underestimated by the patients and/or not properly evaluated and cured by physicians. We believe that the "person-patient" has to know these conditions and that the "person-physician" has to identify and cure them exploring the underlined pathophysiological conditions-which can vary from patient to patientstarting with a personalized therapies, accordingly.

Specific work must be planned and organized to make "persons" aware of the importance of estimating and maintaining muscle mass and its metabolical and functional roles, fundamental in both healthy senescent people and patients. Indeed, simple but useful biomarkers such as TST and AMA can be performed routinely at the bedside of CHF patients in the same way as we take blood pressure. Additional evaluations can also be performed by monitoring plasma protein modifications to understand whether a metabolical imbalance is present. Repeated checks of plasma proteins can also monitor the success or failure of therapeutic procedures. Preliminary data suggest that maintaining global and protein metabolism by specific therapy could maintain muscle mass and function of metabolism, helping traditional therapy.
Conflict of interest None.

\section{References}

1. Wolfe EW (2006) The underappreciated role of muscle in health and diseases. Am J Clin Nutr 84:475-482

2. Anker SD, Ponikowski P, Varney S et al (1997) Wasting as independent risk factor for mortality in chronic heart failure. Lancet 349:1050-1053

3. Aquilani R, Pasini E (2008) Nutrition in acute decompensation of patients with acute heart failure syndrome. In: acute heart failure. Springer-Verlag London Limited, Berlin, pp 876-882

4. Aquilani R, Zuccarelli GC, Bairdi P et al (2011) Effects of oral amino acid supplementation on long-term-care-acquired infection in elderly patients. Arch Gerontol Geriatr 52(3):e123-e128

5. Rolland Y, Abellan van Kan G, Gillette-Guyonnet S, Vells B (2011) Cachexia versus sarcopenia. Curr Opin Clin Nutr Metab Care 14:15-21

6. Pasini E, Aquilani R, Dioguardi FS, D'Antona G, Gheorghiade M, Taegtmeyer H (2008) Hypercatabolic syndrome: molecular basis and effects of nutritional supplementation with amino acids. Am J Cardiol 101:E11-E15

7. Aquilani R, Opasic C, Dossena M et al (2009) Increased skeletal muscle amino acid release with light exercise in deconditioned patients with heart failure. Am J Coll Cardiol 45(1):158

8. Aquilani R, Opasich C, Verri M et al (2003) Is nutritional intake adequate in chronic heart failure patients? J Am Coll Cardiol 42:1218-1223

9. Volpi E, Kobayashi H, Sheffield-Moore M et al (2003) Essential amino acids are primarily responsible for the amino acid stimulation of muscle protein anabolism in healthy elderly adults. Am J Clin Nutr 78:250-258

10. Nisoli E, Cozzi V, Carubba M (2008) Amino Acids and mitochondrial biogenesis. Am J Cardiol 101:22E-25E

11. Fujita S, Dreyer HC, Drummond MJ et al (2007) Nutrient signaling in the regulation of human muscle protein synthesis. J Physiol 582(Pt 2):813-823

12. Aquilani R, Opasich C, Gualco C et al (2008) Adequate energyprotein intake is not enough to improve nutritional and metabolic status in muscle-depleted patients with chronic heart failure. Eur J Heart Fail 10:1127-1135

13. Layman DK (2009) Dietary guidelines should reflect new understandings about adult protein needs. Nutr Metab 6:12

14. Broqvist M, Dahlstrom U, Larsson J, Nylander E, Permert J (1994) Nutritional assessment and muscle energy metabolism in severe chronic congestive heart failure-effects of long-term dietary supplementation. Eur Heart J 15:1641-1650

15. Ebner N, Werner CG, Doehner W et al (2012) Recent developments in treatment of cachexia: highlights form the 6th cachexia conference. J Cachexia Sarcopenia Muscle 3:45-50

16. Burton LA, Sumukadas D (2010) Optimal management of sarcopenia. Clin Interv Aging 5:217-228 